\title{
Review of $B \bar{B}$ Mixing Results
}

\section{Mourad Daoudi}

Stanford Linear Accelerator Center, MS-78, P.O.Box 4349, Stanford, CA 94309, USA

E-mail: 'daoudi@SLAC.Stanford.EDU,

Abstract: A review of $B \bar{B}$ mixing results at the end of July 1999 is presented. Emphasis is put on recent measurements of $\Delta m_{d}$ and $\Delta m_{s}$. For $\Delta m_{d}$, the new world average is $\Delta m_{d}=0.473 \pm 0.016 \mathrm{ps}^{-1}$. For $\Delta m_{s}$, the new world average $95 \% \mathrm{CL}$ limit is $12.4 \mathrm{ps}^{-1}$, with a sensitivity of $14.2 \mathrm{ps}^{-1}$. Other related results are covered very briefly.

\section{Introduction}

The main motivation for performing $B \bar{B}$ mixing measurements lies in the determination of the $C K M$ matrix element $V_{t d}$, which represents one of the constraints on the Unitarity Triangle.

$V_{t d}$ is accessible experimentally through the box diagrams of figure i by measuring the mass difference $\Delta m_{d}$ in $B_{d}$ mixing. $\Delta m_{d}$ and $V_{t d}$ are
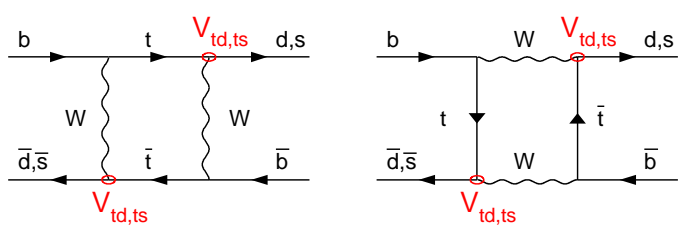

Figure 1: $B \bar{B}$ mixing diagrams.

related by:

$$
\begin{aligned}
\Delta m_{d}= & \frac{G_{F}^{2}}{6 \pi^{2}} m_{b} m_{t}^{2} F\left(\frac{m_{t}^{2}}{m_{W}^{2}}\right) \eta_{Q C D} \\
& \times B_{B_{d}} f_{B_{d}}^{2}\left|V_{t b}^{*} V_{t d}\right|^{2} .
\end{aligned}
$$

Similarly $B_{s}$ mixing provides a measurement of $V_{t s}$

While $\Delta m_{d}$ is measured with very good precision, the determination of $V_{t d}$ is limited by theoretical uncertainties in the decay constant $f_{B_{d}}$ and the bag factor $B_{B_{d}}$. However, in the ratio $\Delta m_{s} / \Delta m_{d}$ most hadronic uncertainties cancel[䁬:

$$
\begin{aligned}
\frac{\Delta m_{s}}{\Delta m_{d}} & =\frac{\eta_{B_{s}} M_{B_{s}} f_{B_{s}}^{2} B_{B_{s}}}{\eta_{B_{d}} M_{B_{d}} f_{B_{d}}^{2} B_{B_{d}}}\left|\frac{V_{t s}}{V_{t d}}\right|^{2} \\
& =(1.14 \pm 0.08)^{2}\left|\frac{V_{t s}}{V_{t d}}\right|^{2} .
\end{aligned}
$$

With $\left|V_{t s}\right| \simeq\left|V_{c b}\right|$, a precise measurement of both $\Delta m_{d}$ and $\Delta m_{s}$ provides a strong constraint on $V_{t d}$. This underlines the importance of $B_{s}$ mixing measurements.

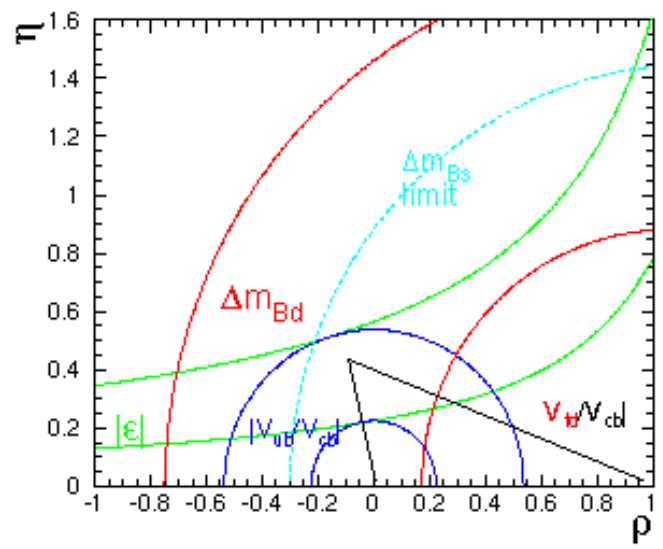

Figure 2: Illustration of the various constraints on the Unitarity Triangle.

In the Wolfenstein parametrization the CKM matrix is defined by four parameters, two of which are quite well known, $\lambda=\sin \theta_{C}=0.2205$ and $A \simeq\left|V_{c b}\right| / \lambda^{2} \sim 0.8$, and two that are not well determined, $\rho$ and $\eta$ whose values define the apex of the Unitarity Triangle. In the $\rho \eta$-plane, $V_{t d}$ is represented by a circle centered at $(1,0)$. It provides one of three constrains on the Unitarity Triangle, the other two being $V_{u b}$ and $\epsilon_{K}$ (figure $\overline{2}$ in. $\Delta m_{d}$ and $\Delta m_{s}$ are given by

$$
\begin{aligned}
& \Delta m_{d} \propto A^{2} \lambda^{6}\left[(1-\rho)^{2}+\eta^{2}\right], \\
& \Delta m_{s} \propto A^{2} \lambda^{4},
\end{aligned}
$$


in terms of $\rho, \eta, \lambda$, and $A$. Note that, unlike $\Delta m_{d}, \Delta m_{s}$ has no dependence on $\rho$ and $\eta$. Therefore, a precise measurement of $\Delta m_{s}$ provides in essence a measure of the product $f_{B_{s}} \sqrt{B_{B_{s}}}$.

Another important aspect is the fact that $B_{s}$ mixing is complementary to the $C P$ violation related measurements that will be performed at $B$ factories in the near future. The measurements of $V_{t d}$ and $\sin 2 \beta$ being essentially orthogonal, they will provide together an excellent constraint on the apex of the Unitarity Triangle in the $B$ system alone. Whereas, measurements of $V_{u b}$ and $\sin 2 \beta$ alone will not be sufficient to provide as good a constraint.

Improvements in lattice calculations continue to be made. For the latest developments in the determination of the decay constant $f_{B_{d}}$ and the bag factor $B_{B_{d}}$, see the talk by $\mathrm{S}$. Hashimoto at this conference 3 . Further details can be found

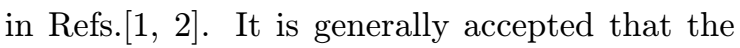
product $f_{B_{d}} \sqrt{B_{B_{d}}}$ lie[[i, $\left[\begin{array}{l}4 \\ -1\end{array}\right]$ in the range $[160,240]$ $\mathrm{MeV}$.

\subsection{Time-integrated mixing measurements}

The probability that a $B^{0}$ meson mix into a $\overline{B^{0}}$ or remain unmixed as a function of proper time is:

$$
\mathcal{P}_{B^{0} \rightarrow \bar{B}^{0}, B^{0}}(t)=\Gamma e^{-\Gamma t}(1 \mp \cos \Delta m t) / 2 .
$$

The time-integrated mixed fraction is given by:

$$
\chi=\frac{(\Delta m / \Gamma)^{2}}{2\left[1+(\Delta m / \Gamma)^{2}\right]} .
$$

At the $\Upsilon(4 S)$ where $B_{d}$ mesons are produced essentially at rest, $\Delta m_{d}$ can extracted from a measurement of $\chi_{d}$. Because $\Delta m_{s}$ is large $\left(1 / \lambda^{2} \approx 20\right.$ times larger than $\left.\Delta m_{d}\right)$, it can be obtained only in a time-dependent mixing measurement $\left(\chi_{s} \sim\right.$ $0.5)$, which makes it rather very challenging.

At the $Z^{0}$ resonance, where all $B$ species are produced, a measurement of $\bar{\chi}=f_{d} \chi_{d}+f_{s} \chi_{s}$ allows the extraction of $f_{s} \equiv f\left(B_{s}\right)$, the fraction of $b$ quarks that hadronize into a $B_{s}$.

\subsection{Time-dependent mixing measurements}

The requirements for a time-dependent mixing measurement are: 1) a precise reconstruction of the proper time of the $B$ meson, 2) a determination of the flavor $b$ or $\bar{b}$ of the $B$ meson both at production (initial-state tag) and at decay (finalstate tag).

A variety of initial-state tags are used, they fall in three categories: 1) vertex and jet charge, high-momentum lepton and kaon, in the opposite hemisphere, 2) fragmentation kaon or pion in the same hemisphere, 3) polarized forward-backward asymmetry. The latter is unique to SLD, it exploits the parity-violating $Z b \bar{b}$ coupling and the presence of a highly polarized electron beam at the SLC.

The most widely used final-state tag is the sign of a high- $P_{t}$ lepton. Other tags include, for example, the sign of a partially or fully reconstructed $D_{s}^{ \pm}$meson, and the dipole charge (see below).

\section{2. $B$ productions fractions}

The LEP B Oscillations Working Group [י, has compiled a new value for $\bar{\chi}=0.1186 \pm 0.0048$. Furthermore, with the inclusion of recent $\Delta m_{d}$ measurements by CLEO and CDF, mentioned below, a new world average value for $\chi_{d}$ was derived: $\chi_{d}=0.176 \pm 0.009$.

A measurement of the charged $B$ branching ratio made by DELPHI increase of the $B_{u}$ production fraction: $f\left(B_{u}\right) \equiv$ $f\left(B_{d}\right)=(40.5 \pm 1.2) \%$. While, the average $b$ baryon production fraction has decreased slightly: $(9.5 \pm 2.0) \%$. As a result, the new $B_{s}$ production fraction becomes:

$$
f\left(B_{s}\right)=(9.6 \pm 1.3) \% .
$$

This value is in good agreement with a separate determination using the measurement of the branching ratio $\mathcal{B}\left(b \rightarrow B_{s}\right) \times \mathcal{B}\left(B_{s} \rightarrow D_{s}^{-} l^{+} \nu X\right)$ which yields: $f\left(B_{s}\right)=(9.7 \pm 2.3) \%$. The new value of $f\left(B_{s}\right)$ is somewhat smaller compared to that evaluated a year ago at the ICHEP'98 conference: $(10.8 \pm 1.3) \%[5]$. This will have the effect of reducing one's sensitivity to $\Delta m_{s}$, compared to one's previous estimate assuming a larger value of $f\left(B_{s}\right)$.

\section{3. $B_{d}$ mixing measurements}

Over the last year there have been two new measurements of $\Delta m_{d}$ : one from CLEO and the other 
from CDF.

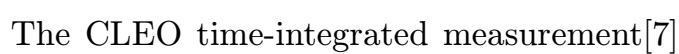
is based on a 4.2 Million $B \bar{B}$ event sample. The final-state $b$ flavor is tagged using the sign of a slow pion in the partially-reconstructed decay chain: $\bar{B}_{d}^{0} \rightarrow D^{*+} \pi^{-}\left(\rho^{-}\right), D^{*+} \rightarrow D^{0} \pi_{s}^{+}$. The initial-state is tagged with a high-momentum lepton in the rest of the event. A value of $\chi_{d}=$ $0.195 \pm 0.026 \pm 0.032$ is obtained, from which the following result for $\Delta m_{d}$ is extracted:

$$
\Delta m_{d}=0.512 \pm 0.053(\text { stat }) \pm 0.032(\text { syst }) \mathrm{ps}^{-1} .
$$

This result is competitive with those from LEP, CDF, and SLD obtained in time-dependent analyses.

At CDF, a new time-dependent measurement

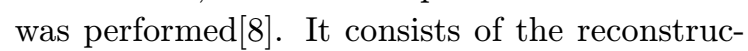
tion of a $\bar{B}_{d}$ decaying to either a $D^{+}$or a $D^{*+}$ :

$$
\begin{aligned}
& \bar{B}_{d}^{0} \rightarrow D^{+} X, \quad D^{+} \rightarrow K^{-} \pi^{+} \pi^{+}, \\
& \bar{B}_{d}^{0} \rightarrow D^{*+} X, D^{*+} \rightarrow D^{0} \pi^{+}, D^{0} \rightarrow K^{-} \pi^{+} .
\end{aligned}
$$

The sign of the $D^{(*) \pm}$ tags the $b$ flavor of the $B_{d}$

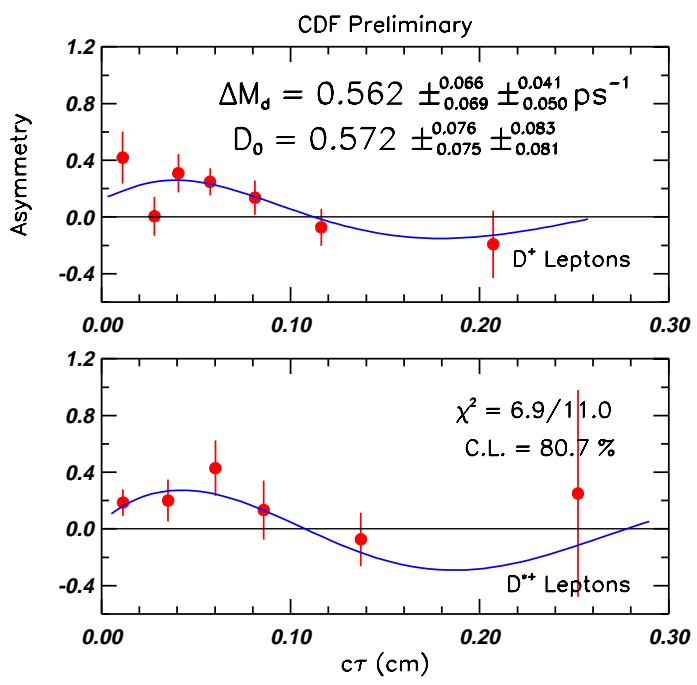

Figure 3: Mixing asymmetry vs. proper time distribution for the $D^{+}$and $D^{*+}$ samples at CDF.

at decay while its flavor at production is given by a high momentum lepton in the opposite hemisphere. A nice feature of this analysis is the fact that $\Delta m_{d}$ is extracted from a maximum likelihood fit in which the tagging dilution factor $\left(D_{0}\right)$ is also fitted. Effectively, the mistag rate is determined directly from the data. figure $\overline{\beta_{1}^{\prime}}$ shows the mixing asymmetry distribution $A(t)=$ $\left(N_{\text {mix }}-N_{\text {unmix }}\right) / N_{\text {tot }}$ as a function of proper time, for the $D^{+}$and $D^{*+}$ samples separately. The combined fit results in:

$$
\Delta m_{d}=0.562 \pm 0.068(\text { stat }){ }_{-0.050}^{+0.041}(\text { syst }) \mathrm{ps}^{-1} .
$$

The value obtained from the fit for the dilution factor is: $D_{0}=0.572 \pm 0.080(\text { stat })_{-0.081}^{+0.083}$ (syst).

figure 4 contains a compilation of about 25 measurements of $\Delta m_{d}$ from the four LEP experiments, CLEO, CDF, and SLD, all very consistent with one another. The new world average value

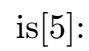

$$
\Delta m_{d}=0.473 \pm 0.016 \mathrm{ps}^{-1} .
$$

Further improvements in $B_{d}$ mixing are expected from CLEO and SLD which have relatively large portions of data not yet analyzed.

\section{4. $B_{s}$ mixing measurements}

Over the last few years, a lot of efforts have been spent on $B_{s}$ mixing. Before reviewing some of the recent analyses, let us mention the first experimental limits on the ratio $\Delta \Gamma_{s} / \Gamma_{s}$ which provide indirect upper limits on $\Delta m_{s}$. Unlike $B_{d}$, recent calculations indicate that for $B_{s}$ the ratio $\Delta \Gamma / \Gamma$ is sizeable $[1001]$. Experimentally, the limits are derived by fitting in a $B_{s}$-enriched sample the proper time distribution to a sum of two exponentials. The best limit comes from DELPHI [1]

$$
\Delta \Gamma_{s} / \Gamma_{s}<0.42 \text { at } 95 \% \text { CL. }
$$

Limits on $\Delta \Gamma_{s} / \Gamma_{s}$ were also set by L3 and CDF. Though the above limit does not provide a strong constraint on $\Delta m_{s}\left(<110 \mathrm{ps}^{-1}\right)$, future improvements are expected as additional and perhaps improved measurements are made.

Three ingredients are required for a $\Delta m_{s}$ measurement, besides a large data sample. They are a high $B_{s}$ enrichment $\left(f_{s}\right)$, a small mistag rate $(\eta)$, and a good proper time resolution $\left(\sigma_{t}\right)$. This is illustrated by the following expression which gives the statistical significance to a $\Delta m_{s}$

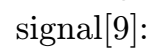

$$
S=\sqrt{N / 2} f_{s}(1-2 \eta) \exp \left[-\left(\Delta m_{s} \sigma_{t}\right)^{2} / 2\right] .
$$

$N$ is the total number of $B$ decays in the sample. The proper time resolution can be expressed 


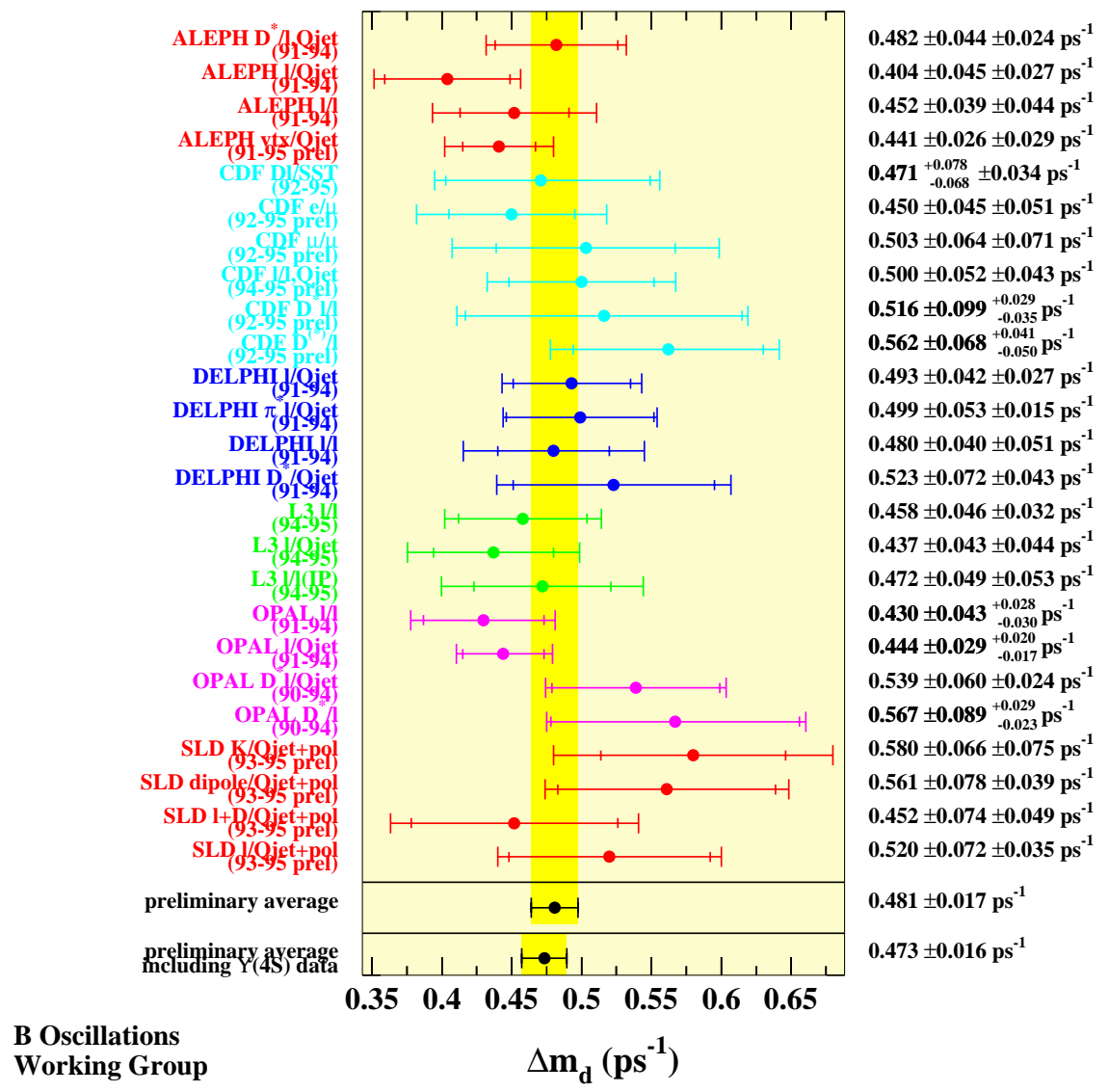

Figure 4: Measurements of $\Delta m_{d}$.

as: $\sigma_{t}^{2}=\left(\sigma_{l} / \beta \gamma c\right)^{2}+t^{2}\left(\sigma_{\beta \gamma} / \beta \gamma\right)^{2}$, where $\sigma_{l}$ is the decay length resolution and $\sigma_{\beta \gamma}$ is the boost resolution. Clearly, for a large value of $\Delta m_{s}$, all the sensitivity is at very short proper time, and the decay length resolution is the dominant term there.

A widely used approach to reconstruct a $B_{s}$ meson and tag its final-state $b$ flavor is to intersect a high- $P_{t}$ lepton with a downstream vertex which may be a fully- or partially-reconstructed $D_{s}^{ \pm}$meson. Sometimes the $B_{s}$ fraction in the sample is enhanced by requiring that a kaon or a $\phi$ meson be present in this vertex.

The extraction of a $\Delta m_{s}$ limit relies on the so-called Amplitude Fit method [9i], where the (1$\left.\cos \Delta m_{s} t\right)$ term in the above expression for the time-dependent mixed probability is replaced by $\left(1-A \cos \Delta m_{s} t\right)$. Values of the amplitude parameter $A$ are scanned for each trial value of $\Delta m_{s}$ in a maximum likelihood fit. At the true mixing frequency, the amplitude $A=1$, and is $=0$ for all other values of $\Delta m_{s}$. The $95 \%$ CL limit corresponds to the smallest value of $\Delta m_{s}$ for which the measured amplitude and its error satisfy the relation: $A+1.645 \sigma_{A}=1$. Similarly, the sensitivity of a given measurement corresponds to the smallest value of $\Delta m_{s}$ for which $1.645 \sigma_{A}=1$ holds.

A total of eleven analyses have been performed by ALEPH, CDF, DELPHI, OPAL, and SLD. This summer, OPAL and DELPHI have produced updates on their $B_{s}$ mixing results. These are described below. For completeness, important results from other experiments are also reviewed.

\subsection{ALEPH inclusive lepton analysis}

This is the most sensitive $B_{s}$ mixing measurement to date [1 $\left.{ }_{2}^{1}\right] . \quad B_{s}$ mesons are reconstructed as the intersection of a high- $P_{t}$ lepton and an inclusive $D$ vertex. The final-state flavor is given 
by the lepton sign, while the initial-state tag relies on vertex charge or a high-momentum lepton in the opposite hemisphere, or a fragmentation kaon in the decay hemisphere. A total of 33,023 decays are selected. The decay length resolution is modeled by a double-Gaussian distribution with a core $(82 \%) \sigma_{l}=280 \mu \mathrm{m}$ and a tail $(18 \%) \sigma_{l}=1060 \mu \mathrm{m}$. The relative boost resolution is estimated by $\sigma_{\beta \gamma} / \beta \gamma=7.1 \%$ in the core $(72 \%)$ and $\sigma_{\beta \gamma} / \beta \gamma=21 \%$ in the tails (28\%). figure 15.1 's shows the amplitude vs. $\Delta m_{s}$ distribution obtained in this analysis. The derived limit is: $\Delta m_{s}>9.5 \mathrm{ps}^{-1}$ at $95 \% \mathrm{CL}$, with a sensitivity of $9.6 \mathrm{ps}^{-1}$. Combined with two other analy-

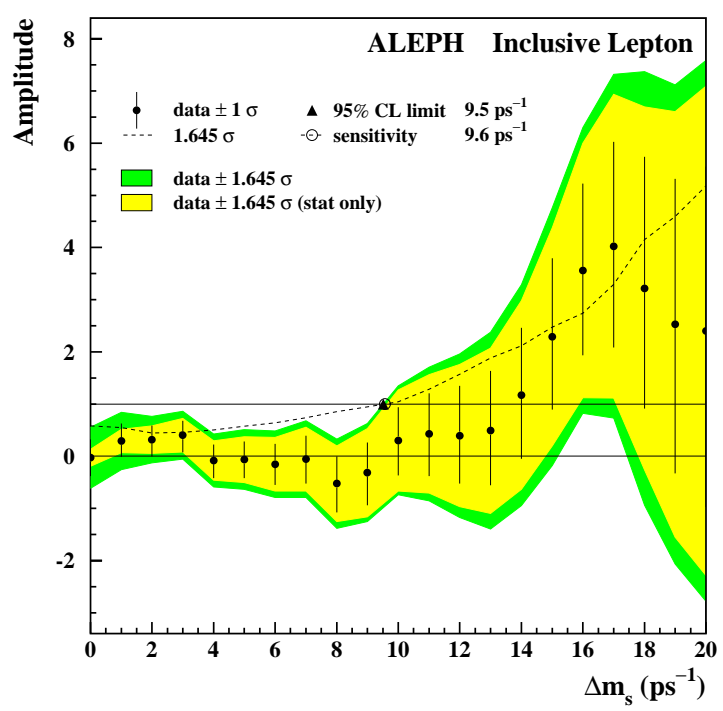

Figure 5: Amplitude distribution for ALEPH's inclusive lepton analysis.

ses $\left(D_{s} l\left[\left[_{1}^{1} \overline{3}_{-1}^{\eta}\right]\right.\right.$ and $D_{s} h\left[\left[_{1}^{1} \overline{4}_{1}^{\prime}\right]\right)$, ALEPH's sensitivity becomes $10.6 \mathrm{ps}^{-1}$ and the overall limit is:

$$
\Delta m_{s}>9.6 \mathrm{ps}^{-1} \text { at } 95 \% \mathrm{CL} \text {. }
$$

\subsection{OPAL inclusive lepton update}

OPAL uses a rather sophisticated technique to reconstruct in $3-\mathrm{D}$ a $B_{s}$ vertex that contains a high- $P_{t}$ lepton [1 ${ }_{1}$. The final-state is tagged with the lepton sign, while the initial-state is given by either jet charge or a high- $P_{t}$ lepton in the opposite hemisphere. In this update, OPAL included their 1995 data and made substantial improvements to their jet charge technique. The final analysis is based on 47,109 single-lepton and
6,031 di-lepton events. The amplitude vs. $\Delta m_{s}$ distribution is shown in figure $\overline{6}^{\prime}$. The sensitivity

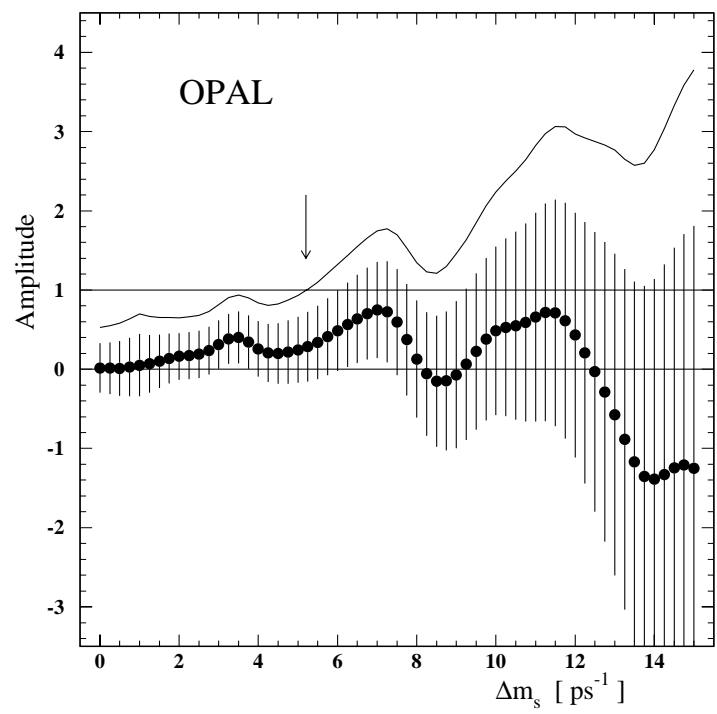

Figure 6: Amplitude distribution for OPAL's inclusive lepton analysis.

is $7.0 \mathrm{ps}^{-1}$ and the limit is:

$$
\Delta m_{s}>5.2 \mathrm{ps}^{-1} \text { at } 95 \% \mathrm{CL} .
$$

\subsection{DELPHI exclusive $B_{s}$ and $D_{s}^{ \pm} h^{\mp}$ up- date}

DELPHI has made the first attempt at an exclusive $B_{s}$ reconstruction [1] ${ }_{1}$. This approach has the advantage of an excellent proper time resolution, with the $B_{s}$ boost being determined exactly. The following decay channels are utilized:

$$
\begin{aligned}
& B_{s} \rightarrow D_{s}^{-} \pi^{+}\left(a_{1}^{+}\right), \\
& B_{s} \rightarrow \bar{D}^{0} K^{-} \pi^{+}\left(a_{1}^{+}\right),
\end{aligned}
$$

where the $D_{s}^{-}$and $\bar{D}^{0}$ are reconstructed in 6 and 2 decay modes, respectively. From the full data sample corresponding to 3.6 Million hadronic $Z$ 's, 11 fully- and 33 partially-reconstructed $B_{s}$ decays are selected with a corresponding $B_{s}$ purity of $70 \%$ and $45 \%$, respectively. With such a small efficiency this analysis by itself has no sensitivity to $\Delta m_{s}$. However, because of its excellent proper time resolution it provides a non-negligible contribution at large $\Delta m_{s}$ values.

DELPHI choose to combine it with the more inclusive $D_{s}^{ \pm} h^{\mp}\left(D_{s} \rightarrow \phi \pi, K^{*} K\right)$ analysis [1] $\left.{ }_{1}\right]$ 
where 2953 candidates are selected with a $B_{s}$ purity of $40 \%$. The overall sensitivity so obtained is $3.2 \mathrm{ps}^{-1}$ and the $95 \% \mathrm{CL}$ limit is $4.0 \mathrm{ps}^{-1}$.

\subsection{DELPHI $D_{s}^{ \pm} l^{\mp}$ update}

This is overall the second most-sensitive $B_{s}$ mixing analysis[1] 1]. A total of 436 decays with a fully-reconstructed $D_{s}$ are selected in the decay modes $D_{s}^{+} \rightarrow \phi \pi^{+}, K^{*} K,^{+}, \phi l^{+} \nu$. Another 441 candidates with a partially-reconstructed (missing $\left.\gamma / \pi^{0}\right) D_{s}^{+} \rightarrow \phi l^{+} X$ are also selected. The $B_{s}$ content in the two sub-samples is estimated to be $230 \pm 18$ and $41 \pm 12 B$ s's, respectively. Clearly, the $D_{s}$ sign provides the final-state tag, while a complex initial-state package including 9 discriminant variables gives the initial-state $b$ flavor with a $78 \%$ purity. The decay length resolution in this analysis is parametrized with a core (86\%) Gaussian with $\sigma_{l}=220 \mu \mathrm{m}$ and a tail (14\%) Gaussian with $\sigma_{l}=870 \mu \mathrm{m}$. Whereas, the boost resolution is characterized by a $5.4 \%$ relative residual in the core $(78 \%)$ and $17.2 \%$ in the tails $(22 \%)$. The $95 \%$ CL limit extracted from this analysis is

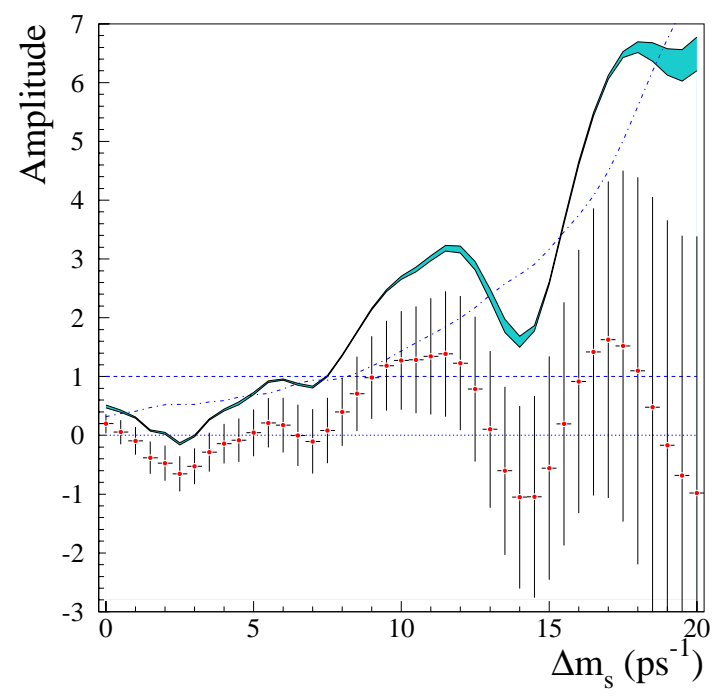

Figure 7: Amplitude distribution for DELPHI's $D_{s} l$ analysis.

$7.4 \mathrm{ps}^{-1}$ with a sensitivity of $8.2 \mathrm{ps}^{-1}$. This is illustrated by the amplitude vs. $\Delta m_{s}$ distribution shown in figure $\overline{\eta_{r}}$. The combined limit from all the DELPHI analyses is:

$$
\Delta m_{s}>5.0 \mathrm{ps}^{-1} \text { at } 95 \% \mathrm{CL}
$$

with an overall sensitivity of $9.7 \mathrm{ps}^{-1}$.

\subsection{CDF $\phi l / l$ analysis}

Using their di-lepton trigger data, $\mathrm{CDF}\left[1 \mathbf{1}_{1}^{-}\right]$perform a partial reconstruction of the $B_{s}$ in the decay chain $B_{s} \rightarrow D_{s} l \nu X \nu \rightarrow \phi l \nu X$. A sample of 1068 candidates is selected with a $B_{s}$ purity of $61 \%$. A second high-momentum lepton in the opposite hemisphere tags the initial-state flavor. This analysis results in a limit of:

$$
\Delta m_{s}>5.8 \mathrm{ps}^{-1} \text { at } 95 \% \mathrm{CL},
$$

and a sensitivity of $5.1 \mathrm{ps}^{-1}$, as shown by the amplitude distribution of figure

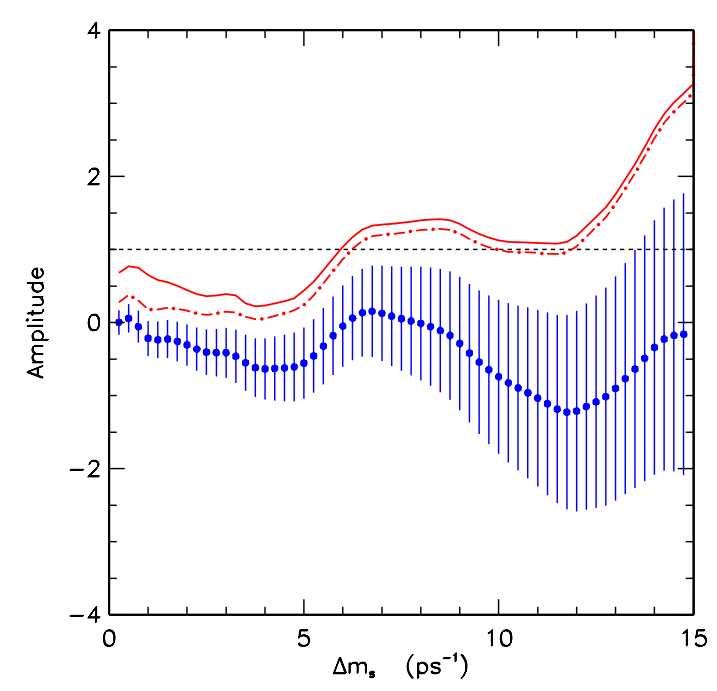

Figure 8: Amplitude distribution for CDF's $\phi l / l$ analysis.

\subsection{SLD $B_{s}$ mixing results}

Using a data sample equivalent to $350 k$ hadronic $Z^{0}$ 's, SLD has performed three $B_{s}$ mixing analyses, referred to as Lepton $+D$, Lepton+Tracks, and Dipole[1] $\left.\overline{1}_{1}\right]$. The number of selected candidates in the three analyses is 2352, 8864, and 8211 , respectively. In the first two, the final-state tag is provided by the high- $P_{t}$ lepton, while in the last one it is given by the dipole charge, defined as the charge difference between the tertiary and secondary vertices multiplied by the distance separating the two vertices. The initial-state tag is given primarily by the polarized forward backward asymmetry, and by jet charge, vertex charge, a high-momentum lepton or kaon in the opposite hemisphere. An excellent decay length resolution 


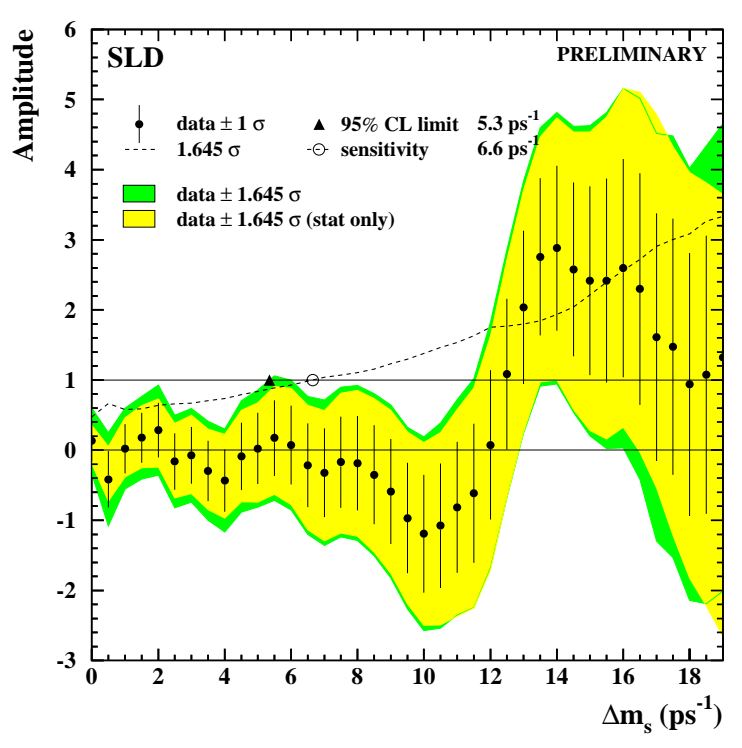

Figure 9: Combined amplitude distribution for the Lepton+D, Lepton+Tracks, and Dipole analyses at SLD.

is achieved at SLD, characterized by a core $(60 \%)$ Gaussian with $\sigma_{l}=105-130 \mu \mathrm{m}$ and a tail (40\%) Gaussian with $\sigma_{l}=330-550 \mu \mathrm{m}$. Good boost resolution is also realized: $\sigma_{\beta \gamma} / \beta \gamma=7-9 \%$ for the core $(60 \%)$ and $\sigma_{\beta \gamma} / \beta \gamma=22-30 \%$ for the tails (40\%). The combined amplitude distribution for the three analyses is shown in figure 9. An overall sensitivity of $6.6 \mathrm{ps}^{-1}$ is obtained, and two separate intervals in $\Delta m_{s}$ are excluded at the $95 \% \mathrm{CL}$ :

$$
\begin{aligned}
\Delta m_{s} & <5.3 \text { and } \\
6.0<\Delta m_{s} & <11.5 \mathrm{ps}^{-1} .
\end{aligned}
$$

\subsection{Summary of $\Delta m_{s}$ results}

In the following, a summary as of the end of July 1999 of all $B_{s}$ mixing results compiled by the LEP B Oscillations Working Group [05] is given. figure $11_{1}^{1}$ gives the measured amplitude and its error at a $\Delta m_{s}$ value of $15 \mathrm{ps}^{-1}$, as well as the sensitivity of each analysis. The world average amplitude at $\Delta m_{s}=15 \mathrm{ps}^{-1}$ is $2.2 \sigma$ away from zero $(1.53 \pm 0.69)$.

The world average amplitude vs. $\Delta m_{s}$ distribution is shown in figure 121 . The overall sensitivity is $14.2 \mathrm{ps}^{-1}$ and the combined limit:

$$
\Delta m_{s}>12.4 \mathrm{ps}^{-1} \text { at } 95 \% \mathrm{CL} .
$$

Note that this limit does not take into account the new smaller value of the $B_{s}$ production fraction, as discussed above. Compared to last year's results at the ICHEP'98 conference $1 \overline{1}^{\prime}$, the $\Delta m_{s}$ limit is unchanged, while the overall sensitivity has improved slightly (up from $13.8 \mathrm{ps}^{-1}$ ). The

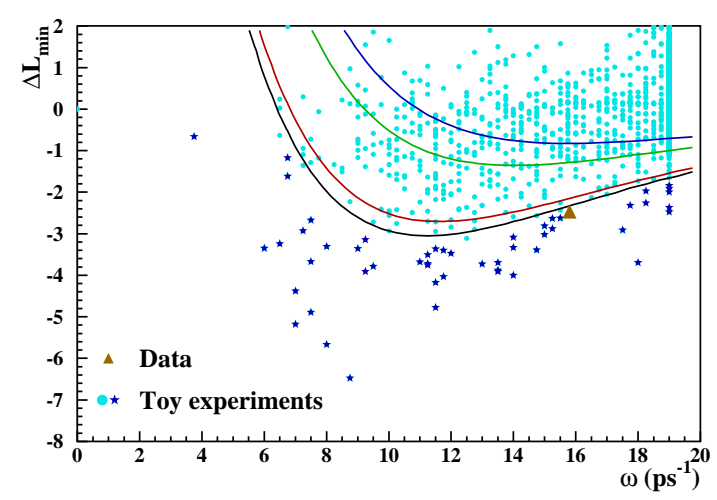

Figure 10: Likelihood profiles for 2000 toy Monte Carlo samples with $\Delta m_{s}=150 \mathrm{ps}^{-1}$. The triangle represents the experimental data as of winter'99.

contribution of the limit on $\Delta m_{s}$ to the determination of CKM matrix is summarized in Ref. [1 $\left.199_{1}^{\prime}\right]$.

The bump seen in the amplitude distribution at around $15 \mathrm{ps}^{-1}$ has been the subject of a lot interest. In a recent contribution, D. Abbaneo and G. Boix [201] proposed a procedure to estimate the probability that the observed structure is due to a pure statistical fluctuation. They ran 2000 fast Monte Carlo experiments with a large value of $\Delta m_{s}\left(150 \mathrm{ps}^{-1}\right)$, each experiment being equivalent to the world sensitivity as of last win-

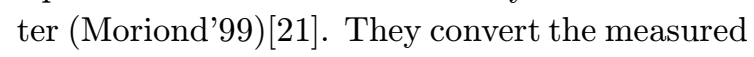
amplitudes into likelihood profiles as a function of $\Delta m_{s}$. From these profiles which are shown in figure 10 10 they estimate the probability that the bump in the data (represented by the triangle in the figure) to originate from a fluctuation in a no-signal sample to be $\sim 3-5 \%$. 


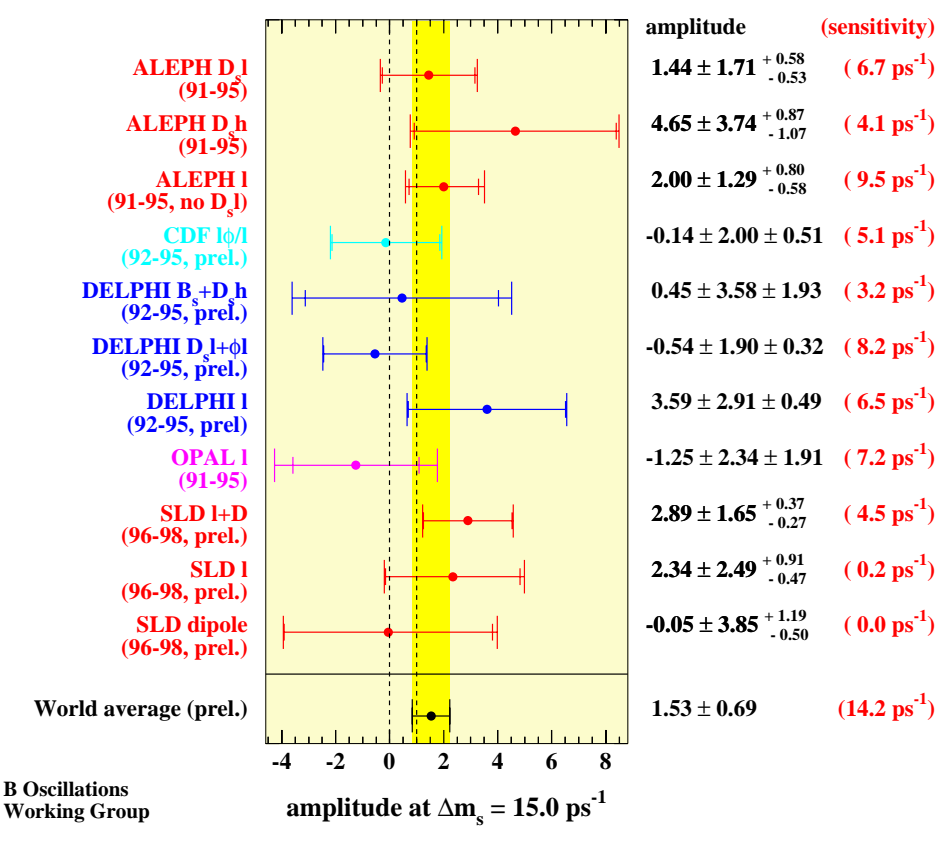

Figure 11: Measured amplitude at $\Delta m_{s}=15 \mathrm{ps}^{-1}$ for each analysis.

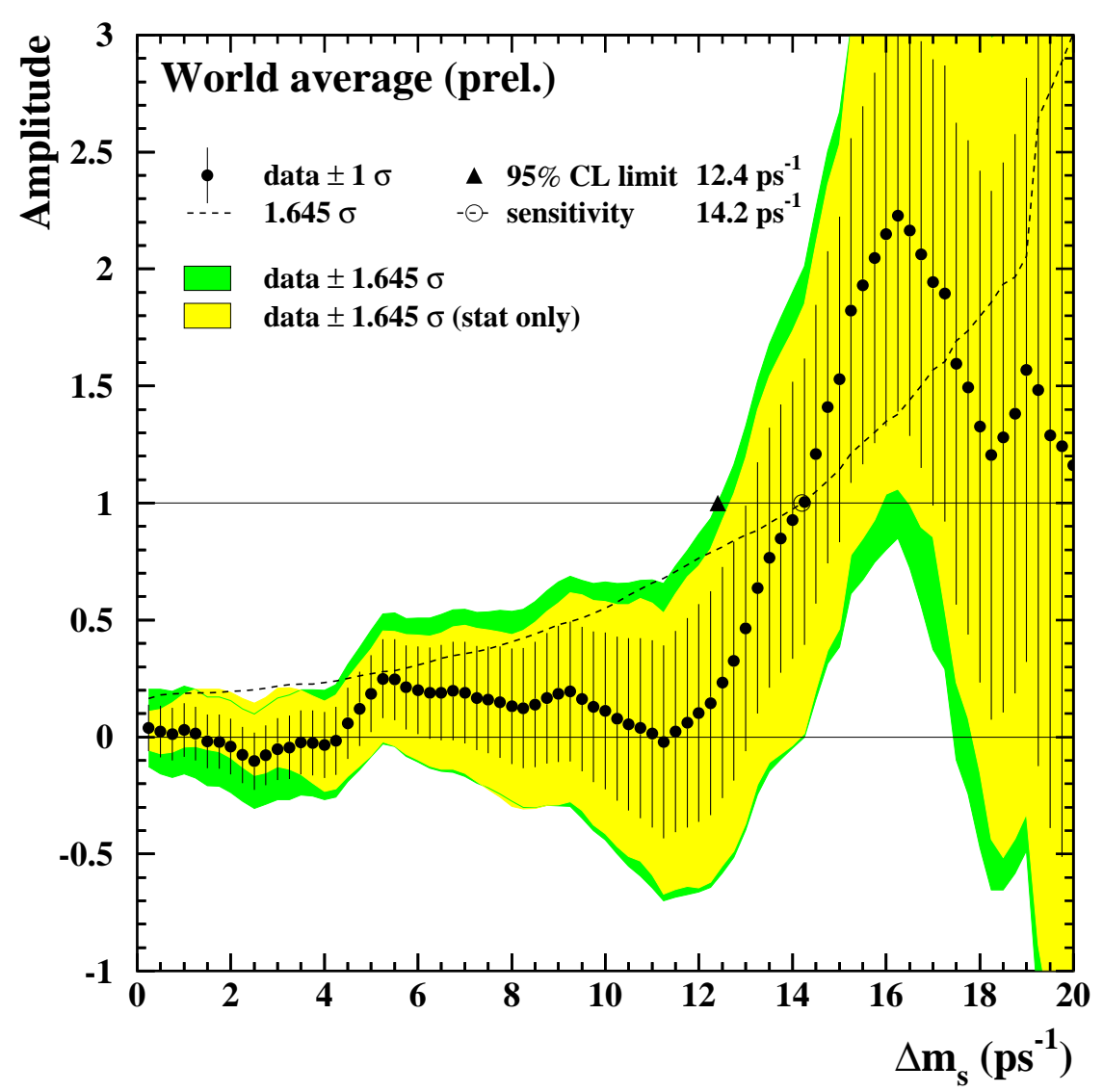

Figure 12: World average amplitude vs. $\Delta m_{s}$ distribution. 


\subsection{SLD status and future prospects}

Final $B_{s}$ mixing results from SLD are expected in the near future. Since Moriond'99, substantial improvements have been achieved, they come from the following three sources:

- Improved tracking resolution.

- Improved charge dipole reconstruction.

- Addition of two new analyses.

The design resolution with the pixel vertex detector VXD3 has been realized. It is characterized by a track impact parameter resolution of $7.8 \mu \mathrm{m}$ in the $r \phi$-plane and $9.7 \mu \mathrm{m}$ in the $r z$ plane. Furthermore, the precise location of the micron-size SLC beam spot is determined with an uncertainty of $4 \mu \mathrm{m}$.

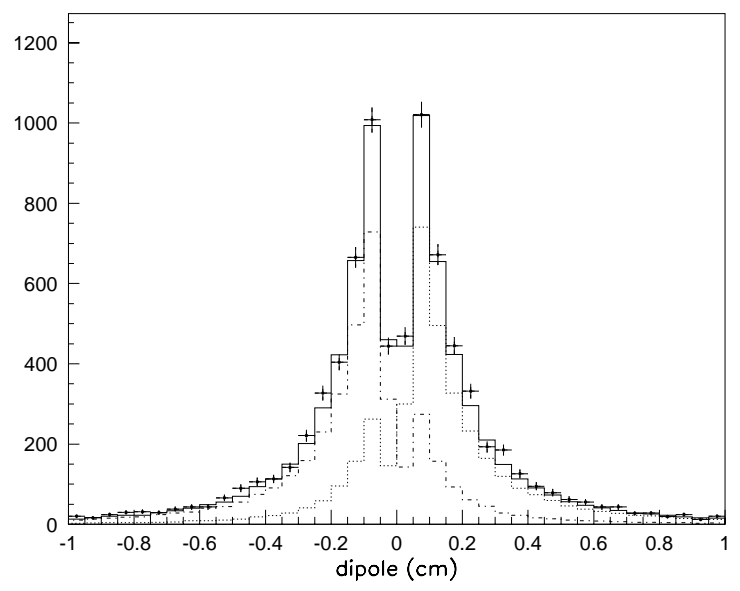

Figure 13: SLD's dipole charge distribution for data (points) and Monte Carlo (histogram). The dotted and dashed histograms represent the $b$ - and $\bar{b}$ components, respectively.

Improvements in the SLD's topological vertexing algorithm[22in have resulted in a significantly better dipole tag purity (80\% compared to $73 \%$ previously). The dipole tag, which is unique to SLD, exploits the charge flow in the cascade decay $b \rightarrow c$, and is made possible due to the ability to reconstruct (topologically) wellseparated $B$ and $D$ vertices. The dipole charge is reconstructed as the charge difference between the $B$ and $D$ vertices multiplied by the distance separating them. It is shown in figure $1 \overline{1}$, where the $b$-quark and $\bar{b}$-quark components are represented by the dotted and the dashed histograms, respectively.
One of the two new analyses at SLD, called the $D_{s}+$ Tracks analysis, relies on the exclusive reconstruction of a $D_{s}^{+}$in the $\phi \pi^{+}$and $K^{* 0} K^{+}$ decay modes. It provides both a high $B_{s}$ purity of $33 \%$, and an excellent decay length resolution $\sigma_{l}=46 \mu \mathrm{m}$ in the core $(60 \%)$ and $\sigma_{l}=158 \mu \mathrm{m}$ in the tails (40\%).

In the second new SLD analysis, called the Lepton + Kaon analysis, final-states containing an opposite sign lepton - kaon pair are selected. It is aimed primarily at isolating the semileptonic decays: $B_{s} \rightarrow D_{s}^{* *-} l^{+} \nu, D_{s}^{* *-} \rightarrow K^{-} \bar{D}^{(*) 0}$. Decays of $B^{ \pm}$and $B_{d}$ mesons produce lepton kaon pairs with the same sign and are therefore suppressed. Thus, resulting in an enhancement of the $B_{s}$ purity. This analysis is also sensitive to the decays $B_{s} \rightarrow D_{s}^{-} l^{+} \nu, D_{s}^{-} \rightarrow \phi X$, $\phi \rightarrow K^{-} K^{+}$, in particular with the additional requirement of a second kaon in the $B_{s}$ vertex. The $B_{s}$ purity that is achieved is $26 \%$, while a very good decay length resolution is also obtained with $\sigma_{l}=71 \mu \mathrm{m}$ in the core $(60 \%)$ and $\sigma_{l}=330 \mu \mathrm{m}$ in the tails (40\%). Both these new analyses have the disadvantage of a small overall efficiency. They have a small sensitivity to $\Delta m_{s}$, but provide a substantial contribution at high values of $\Delta m_{s}$ when combined with the other SLD analyses.

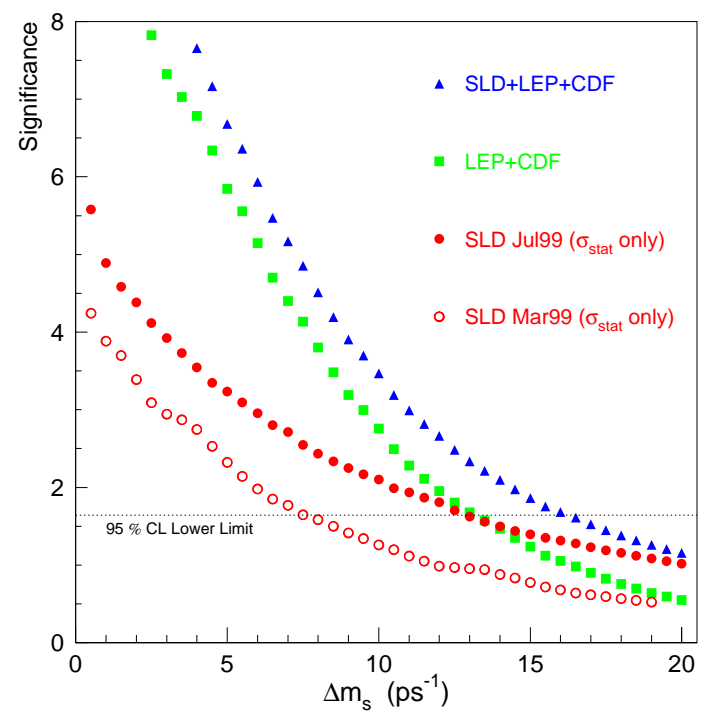

Figure 14: Estimated improvement in SLD's sensitivity and its impact on the world average.

With these improvements, SLD's overall $\Delta m_{s}$ 
sensitivity is estimated to increase to $\sim 13 \mathrm{ps}^{-1}$, and to dominate the world average for $\Delta m_{s}>$ $13 \mathrm{ps}^{-1}$. The combined LEP $+\mathrm{CDF}+\mathrm{SLD}$ sensitivity becomes $\sim 16 \mathrm{ps}^{-1}$. This is illustrated in figure i14'.

Several new experiments $\left[2 \overline{2} \overline{3}_{-1}^{1}, \overline{2} \overline{4}_{-1}^{1}, \overline{2} \overline{2} \overline{5}_{1}^{\prime}\right]$ specifically geared for $B$ physics, $C P$ violation in particular, are planned for the next decade. Their expected reach for $B_{s}$ mixing is shown in figure 115. It is anticipated that CDF will be the first experiment to measure $\Delta m_{s}$, with a sensitivity of $40 \mathrm{ps}^{-1}$. Note that SLD's reach could have been extended to $\Delta m_{s}=20 \mathrm{ps}^{-1}$. However, the run extension that was requested in order to achieve that was not approved due to lack of funding.

Bs Mixing Reach

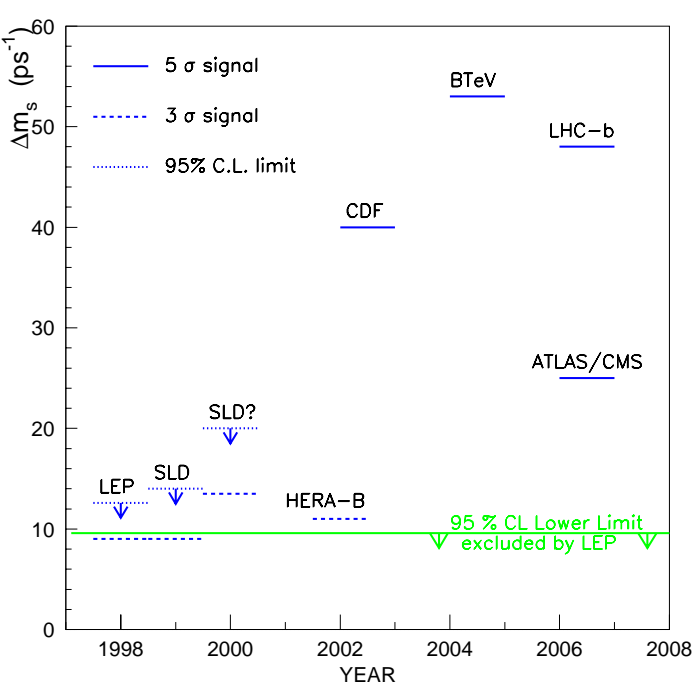

Figure 15: Expected $B_{s}$ mixing reach for futuregeneration $B$ physics experiments.

\section{Conclusions}

We have presented a review of $B$ mixing results as of the end of July 1999 . For $B_{d}$ mixing, with a new measurement from CDF, the new world average for $\Delta m_{d}$ becomes:

$$
\Delta m_{d}=0.473 \pm 0.016 \mathrm{ps}^{-1} .
$$

In $B_{s}$ mixing, updates from OPAL and DELPHI were produced recently. The new world average average limit is:

$$
\Delta m_{s}>12.4 \mathrm{ps}^{-1} \text { at } 95 \% \mathrm{CL},
$$

and the new world average sensitivity is $14.2 \mathrm{ps}^{-1}$. This, without taking into account the smaller value of the $B_{s}$ production fraction, which is evaluated by the LEP B Oscillations Working Group to be:

$$
f\left(B_{s}\right)=(9.6 \pm 1.3) \% .
$$

A relatively significant $(\sim 2 \sigma)$ bump persists in the amplitude spectrum at around $\Delta m_{s}=$ $15 \mathrm{ps}^{-1}$. An attempt at estimating the probability that this may be due to a statistical fluctuation was performed by G. Abbaneo and G. Boix (using winter'99 data). Their result is a probability of $\sim 3-5 \%$.

We have also seen the first attempts at constraining $\Delta m_{s}$ from above, by setting a lower limit on the ratio $\Delta \Gamma_{s} / \Gamma_{s}$. The best limit comes from DELPHI:

$$
\Delta \Gamma_{s} / \Gamma_{s}<0.42 \text { at } 95 \% \text { CL. }
$$

In the near future, final results from SLD are expected, with substantial improvements coming from a better tracking resolution, an improved dipole charge reconstruction, and the inclusion of two new analyses. As of this writing, a partial update from SLD [26 sented at the Lepton-Photon'99 conference. In fact the world average $\Delta m_{s}$ limit and sensitivity

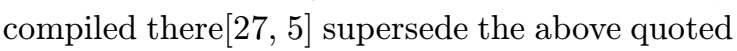
values.

In the longer term, many $B$ physics experiments are planned well into the next decade. With a $\Delta m_{s}$ reach extending over a very wide range, a precise measurement of $\Delta m_{s}$ will be performed by these experiments.

\section{Acknowledgments}

I would like to thank my colleagues David Jackson, John Jaros, and Stéphane Willocq for very fruitful discussions. 


\section{References}

[1] J.M. Flynn, C.T. Sachrajda, [heplat/9710057].

Also in Heavy Flavours II, pp. 402-452 (World

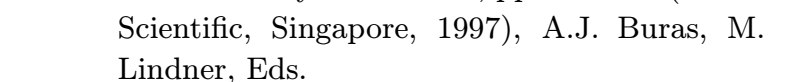

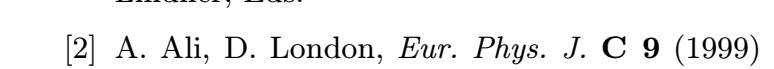

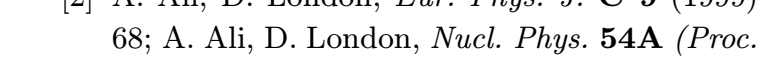
Suppl.) (1997) 297 .

[3] S. Hashimoto, "Summary of lattice results for decay constants, mixing, etc.", in these proceedings.

[4] "The BABAR Physics Book", P.F. Harrison, H.R. Quinn, Eds., [SLAC-R-504] (1998).

[5] LEP B Oscillations Working Group, see http://www.cern.ch/LEPBOSC/ (and links/references therein).

[6] DELPHI Collaboration, [DELPHI 99-104, CONF 291].

[7] CLEO Collaboration, see http://www.lns.cornell.edu/public/CLEO /analysis/results/B-mixing/.

[8] CDF Collaboration, see http://www-cdf.fnal.gov/physics/new /bottom/cdf4526/cdf4526.html.

[9] H.G. Moser, A. Roussarie, Nucl. Inst. Meth. A 384 (1997) 491.

[10] See for example M. Beneke et al., iPhys. Lett. $\mathbf{B} \overline{459}(\overline{1999)} 6 \overline{1}$.

[11] DELPHI Collaboration, [DELPHI 99-109, CONF 296].

[12] ALEPH Collaboration, iEur. Phys. J. C : - _ _ (1999) 553 ; [hep-ex/9811018].

[13] ALEPH Collaboration, 'Phys. Lett. B $\overline{\mathbf{3} 7 \overline{7}}$ (1996) $20 \bar{i}$

[14] ALEPH Collaboration, Eur. Phys. J. C C ' - - -

[15] OPAL Collaboration, [CERN-EP-99-085], June 1999, 28pp., [hep-ex/9907061].

[16] CDF Collaboration, 'L ! - - - -3576

[17] J. Thom, SLD Collaboration, Proceedings of Beauty'99, Bled, Slovenia, June 1999, to appear.

[18] F. Parodi, Proceedings of the 29th International Conference on High Energy Physics, Vancouver 1998, vol. 2, pp. 1148-1154.
[19] S. Plaszczynski, "Overall determination of the CKM Matrix", in these proceedings.

See also:

F. Parodi, P. Roudeau, A. Stocchi, [hepex/9903063]; S. Mele, 'Phys. Rev. D_59 (1999)' $113011_{1}^{\prime}$

[20] G. Boix, D. Abbaneo, 'J. High Energy Phys. 08' (1999) 004, [hep-ex/9909033].

[21] LEP B Oscillations Working Group, "Combined Results on $B^{0}$ Oscillations: Results from Winter 1999 Conferences", [LEPBOSC 99/1], June 1999

[22] D.J. Jackson, Nucl. Inst. Meth. A 388 (1997) 247.

[23] E. Gerndt, "Status of HERA-B", in these proceedings.

[24] R. Jesik, "b-physics potential of Tevatron Run II", in these proceedings.

[25] N. Harnew, "Prospects for LHCb, BTeV, ATLAS, CMS", in these proceedings.

[26] SLD Collaboration, [SLAC-PUB-8225], Aug. 1999, 25pp.

[27] G. Blaylock, Proceedings of Lepton-Photon'99, Stanford, CA, August 1999, to appear. 